\title{
EJACULATION DISORDERS
}

\section{Discontinuation of Dapoxetine Treatment in Patients With Premature Ejaculation: A 2-Year Prospective Observational Study}

Hyun Jun Park, MD, PhD, Nam Cheol Park, MD, PhD, Tae Nam Kim, MD, Seung Ryong Baek, MD, Kyung Min Lee, MS, and Sangmin Choe, MD, $\mathrm{PhD}^{2}$

\begin{abstract}
Introduction: Although dapoxetine is the only oral pharmacologic agent approved for the treatment of premature ejaculation (PE) and is very effective, the discontinuation rate is high.

Aim: To assess the discontinuation rate of patients with PE and the reasons for discontinuation in real-world practice.

Methods: In total, 182 consecutive patients were enrolled. Type of PE, self-estimated intravaginal ejaculation latency time, and medical history were evaluated in all patients who also completed the erectile function domain of the International Index of Erectile Function (IIEF). Visits were scheduled 1, 3, 6, 12, and 24 months after initiation of therapy; treatment status and the reasons for discontinuation in those who did discontinue were checked. The relations of discontinuation rates were compared with various parameters and the time to discontinuation after treatment commencement.

Results: Of all patients, $9.9 \%$ continued treatment to 2 years. The cumulative discontinuation rates at 1, 3, 6, 12, and 24 months were $26.4 \%, 61.6 \%, 79.1 \%, 87.3 \%$, and $90.1 \%$, respectively. Moreover, $79.1 \%$ of all patients discontinued treatment within 6 months. After 12 months, the discontinuation rate decreased sharply. The reasons for discontinuation were cost (29.9\%), disappointment that PE was not curable and that dapoxetine was required every time sexual intercourse was contemplated (25\%), side effects (11.6\%), perceived poor efficacy $(9.8 \%)$, a search for other treatment options (5.5\%), and unknown (18.3\%). Patients with acquired PE (vs lifelong PE), with intravaginal ejaculation latency time longer than 2 minutes before treatment, on phosphodiesterase type 5 inhibitors, and with IIEF erectile function scores lower than 26 tended to discontinue early and thus exhibited high dropout rates.

Conclusion: The treatment discontinuation rate of dapoxetine was very high. The main reasons for discontinuation were the cost and disappointment that treatment was required every time adequate sexual function was required. Park HJ, Park NC, Kim TN, et al. Discontinuation of Dapoxetine Treatment in Patients With Premature Ejaculation: A 2-Year Prospective Observational Study. Sex Med 2017;5:e99-e105.

Copyright (c) 2017, The Authors. Published by Elsevier Inc. on behalf of the International Society for Sexual Medicine. This is an open access article under the CC BY-NC-ND license (http://creativecommons.org/licenses/by-nc-nd/4.0/).
\end{abstract}

Key Words: Premature Ejaculation; Serotonin Uptake Inhibitors; Compliance

\section{INTRODUCTION}

Premature ejaculation (PE) is one of the most common male sexual dysfunctions. PE decreases sexual satisfaction and the quality of life of patients and their partners. ${ }^{1}$ Recently, the

Received January 14, 2017. Accepted February 28, 2017.

'Department of Urology and Medical Research Institute, Pusan National University Hospital, Pusan National University School of Medicine, Busan, Korea;

${ }^{2}$ Department of Clinical Pharmacology and Therapeutics, Pusan National University Hospital, Busan, Korea

Copyright (c) 2017, The Authors. Published by Elsevier Inc. on behalf of the International Society for Sexual Medicine. This is an open access article under the CC BY-NC-ND license (http://creativecommons.org/ licenses/by-nc-nd/4.0/).

http://dx.doi.org/10.1016/j.esxm.2017.02.003
International Society for Sexual Medicine defined PE is a "male sexual dysfunction characterized by ejaculation that always or nearly always occurs prior to or within $1 \mathrm{~min}$ of vaginal penetration from the time of the first sexual experience (lifelong PE), or a clinically significant reduction in latency time, often to about 3 minutes or less (acquired PE)." ${ }^{2}$ Currently, several treatments for PE are available. Psychological-behavioral pharmacologic therapies, including selective serotonin reuptake inhibitors (SSRIs), tricyclic antidepressants, tramadol, phosphodiesterase type 5 (PDE5) inhibitors, and $\alpha_{1}$-andreoreceptor antagonists; topical anesthetics; and even surgery have been used to treat PE in real-world practice. ${ }^{3}$ Dapoxetine is the first oral pharmacologic agent developed to treat PE and is the only SSRI approved for such treatment in more than 60 countries. ${ }^{4}$ The introduction of dapoxetine was associated with high expectations, given the 
optimal efficacy-safety profiles evident in phase 3 trials. ${ }^{5}$ However, although several clinical studies have confirmed drug efficacy in increasing the intravaginal ejaculation latency time (IELT) and safety, the rate of discontinuation is as high as when taking off-label SSRIs. ${ }^{4,5}$ The discontinuation rate is high compared with those of PDE5 inhibitors in patients with erectile dysfunction (ED). ${ }^{4,6}$

To date, dapoxetine is the only approved medical treatment for PE; no second-line therapy is available in those who do not respond or who refuse to take dapoxetine. Thus, a comprehensive evaluation of factors leading to dropout was needed, especially in a real-practice setting. Therefore, we assessed dapoxetine discontinuation by patients with PE in a clinical setting, and the reasons for such discontinuation, over a follow-up period of 2 years.

\section{METHODS}

\section{Study Design}

This was a 2-year prospective observational study conducted in a single clinical center in accordance with Good Clinical Practice and in conformity with the ethical principles of the Declaration of Helsinki. The local ethics committee approved the study protocol and all patients signed informed consent forms.

At baseline, all patients were asked to self-estimate their IELT using a stopwatch, completed the International Index of Erectile Function erectile function domain (IIEF-EFD), and gave a medical history. The IELT was defined as the time from the start of vaginal intromission to commencement of intravaginal ejaculation. ${ }^{7}$ According to the new definition of the International Society of Sexual Medicine, ${ }^{2}$ lifelong PE was defined as ejaculation that always or nearly always occurred before or within approximately 1 minute of vaginal penetration, commencing at the first sexual experience, and acquired PE was defined as a clinically significant and bothersome decrease in latency time, often to no longer than 3 minutes. The two types of PE have been associated with an inability to delay ejaculation after all or nearly all vaginal penetrations and associated with negative personal consequences, including distress, worry, frustration, and/or avoidance of sexual intimacy.

\section{Subjects}

In total, 182 consecutive patients seeking medical treatment for PE were enrolled. The inclusion criteria were male sex, no history of dapoxetine treatment, age older than 19 years, and involvement in a stable monogamous relationship with a female sexual partner.

Men with the following conditions were excluded: any anatomic penile deformity; spinal cord injury; prior radical prostatectomy; pelvic organ surgery; diagnosis of any sexual disorder other than ED; any uncontrolled psychiatric disorder; any history of a major hematologic, renal, or hepatic abnormality; a history of alcoholism or substance abuse; and/or an organic illness limiting the ability to take SSRIs. Patients did not receive any financial incentive to participate in this observational survey. Concomitant use of any other PE treatment was prohibited during the study period.

\section{Outcome Measurements}

After a 4-week run-in period, patients took dapoxetine $30 \mathrm{mg}$ 1 to 3 hours before planned sexual intercourse. Dose escalation to $60 \mathrm{mg}$ was allowed after 1 month if the $30-\mathrm{mg}$ dose was inadequate. Patients were re-evaluated 1, 3, 6, 12, and 24 months after initiating therapy for treatment status and the reason for discontinuation if they had discontinued. If a patient missed any visit, then he was contacted by telephone or mail to collect the required data. The primary end points were the discontinuation rates and the reasons for discontinuation. We compared the discontinuation rates with various parameters and the interval from commencement to discontinuation.

\section{Statistical Analyses}

We undertook a prospective cohort study. We compared the baseline characteristics of patients continuing and discontinuing treatment using the $\chi^{2}$ test to evaluate categorical variables. A $P$ value less than 0.05 was considered statistically significant. All statistical analyses were performed using SPSS 15.0 for Windows (SPSS Inc, Chicago, IL, USA).

\section{RESULTS}

We consecutively enrolled 182 patients from October 1, 2011 through September 30, 2013. The mean age of all patients was 38.2 years (range $=19-63$ ) and the mean baseline IELT was $61.2 \pm 23.4$ seconds. Of all patients, $9.9 \%$ continued treatment to 2 years. The cumulative discontinuation rates at $1,3,6,12$, and 24 months were $26.4 \%, 61.6 \%, 79.1 \%, 87.3 \%$, and $90.1 \%$, respectively. Moreover, $79.1 \%$ of all patients discontinued treatment within 6 months. After 12 months, the discontinuation rate decreased sharply (Figure 1). Patients with acquired PE (vs lifelong PE), with IELTs longer than 2 minutes before treatment, on PDE5 inhibitors, and with IIEF-EFD scores lower than 26 tended to exhibit high dropout rates at the end of the study (Table 1). The reasons for discontinuation were cost (29.9\%), disappointment that PE was not curable and that dapoxetine was needed whenever sex was planned $(25 \%)$, side effects $(11.6 \%)$, perceived inefficacy $(9.8 \%)$, a search for other treatment options (5.5\%), and unknown (18.3\%; Table 2). The most common side effects included yawning, nausea, dizziness, and headache. No severe side effects, such as self-harming or aggressive behavior, serotonin syndrome, postural hypotension, or syncope, were reported.

\section{DISCUSSION}

Before the introduction of dapoxetine, off-label SSRIs, topical anesthetics, and the narcotic analgesic tramadol were the only medical agents used to treat PE. The discontinuation rates in patients with PE were very high. ${ }^{7,8}$ Salonia et $\mathrm{al}^{7}$ found that up 

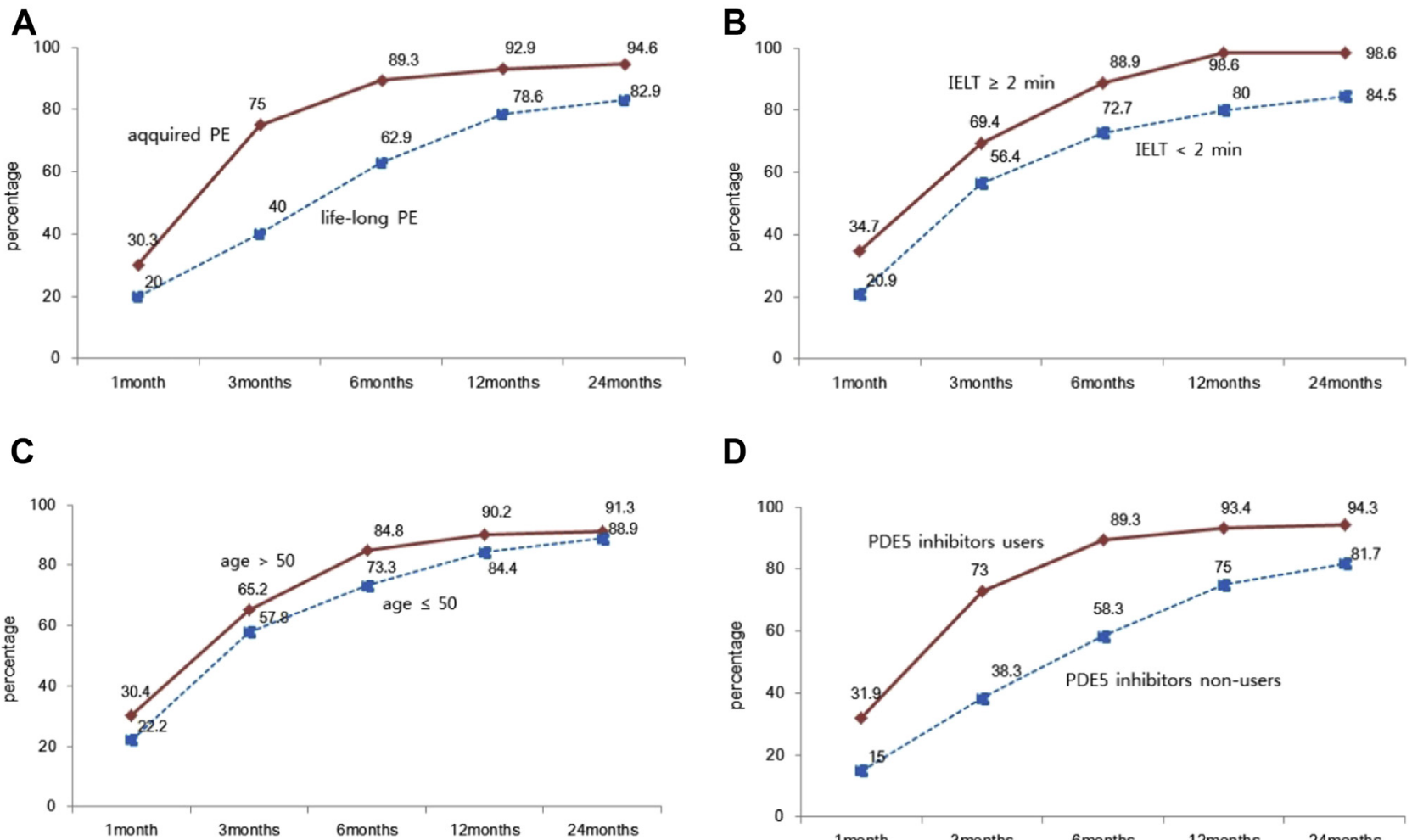

D
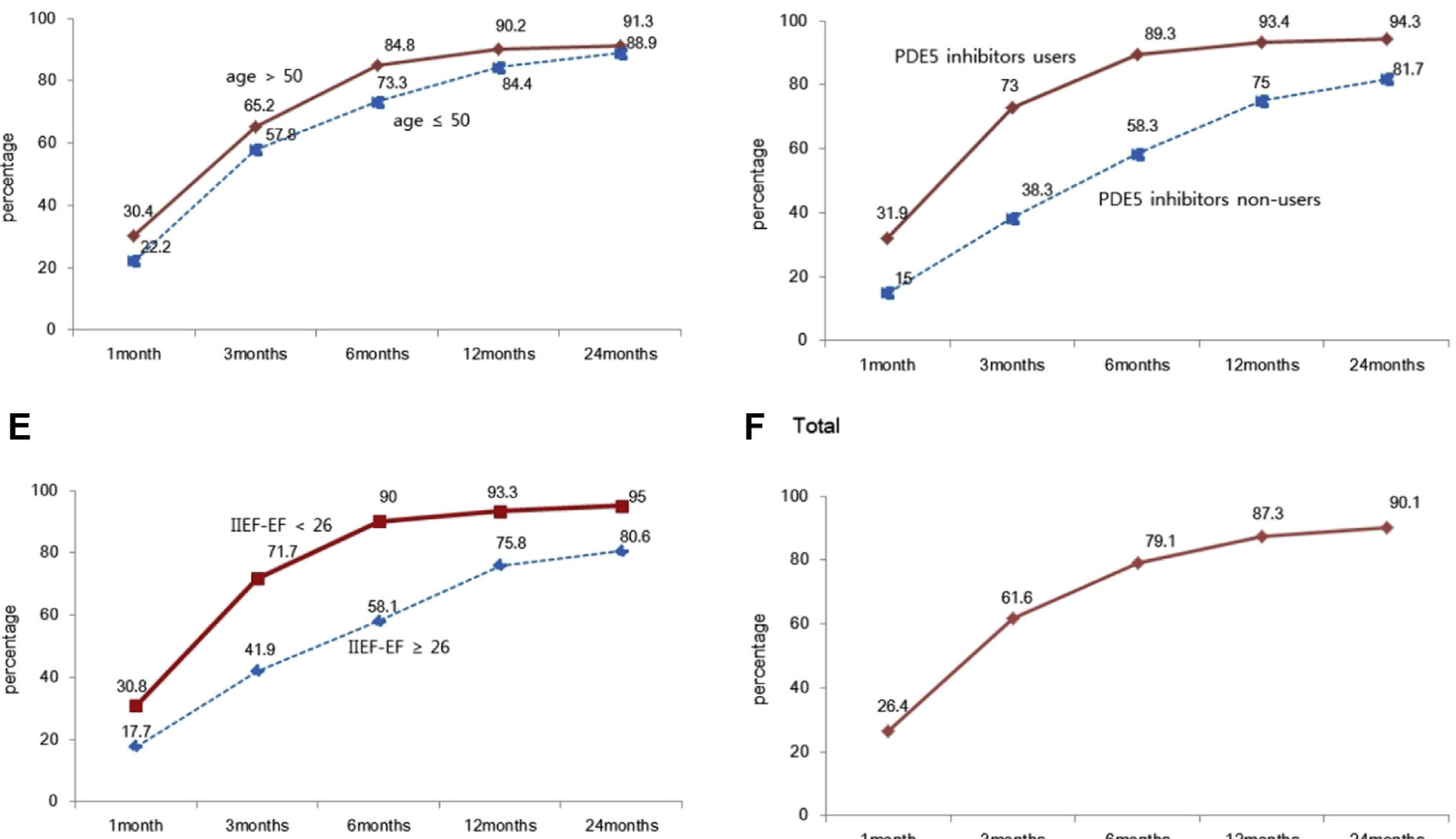

\section{F Total}

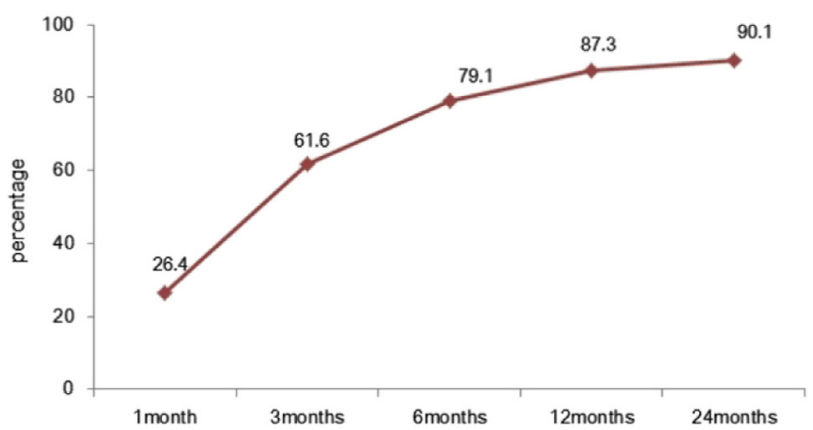

Figure 1. Cumulative discontinuation rates. Panel A shows acquired PE vs lifelong PE. Panel B shows IELT at least 2 vs shorter than 2 minutes. Panel $C$ shows age older than 50 vs no older than 50 years. Panel D shows users of PDE5 inhibitors vs non-users. Panel E shows IIEF-EF scores lower than 26 vs at least 26. Panel F shows total. IIEF-EF = International Index of Erectile Function erectile function domain; PDE5 = phosphodiesterase type 5; PE = premature ejaculation.

to $60 \%$ of patients who were prescribed paroxetine to treat lifelong PE eventually discontinued the drug. In addition, psychological and behavioral therapy and later the "squeeze" technique were associated with high discontinuation rates., 10 Although topical anesthetic agents were effective in the short term, no long-term data have been reported. ${ }^{11}$

Dapoxetine is suited for "on-demand" treatment of PE because the drug is rapidly absorbed and has a short initial half-life. ${ }^{12}$ The other SSRIs require daily dosing, increasing the risk of class treatment-emergent adverse events. Dapoxetine attains a peak plasma concentration approximately 1.5 hours after dosing compared with 6 hours for fluoxetine and 5 hours for paroxetine. $^{13}$ Then, dapoxetine plasma levels decrease rapidly, to attain a concentration only $4 \%$ that of the peak 24 hours after dosing. ${ }^{14}$ Dapoxetine pharmacokinetics is not affected by multiple dosing; the drug does not accumulate to a significant extent. $^{13}$

Several randomized controlled trials have reported on the efficacy and safety of dapoxetine in more than 6,000 patients with $\mathrm{PE}$ in more than 25 countries. ${ }^{15-18}$ Integrated analysis of these phase 3 trials showed a significant increase in the geometric mean IELT. ${ }^{5}$ In addition, dapoxetine improved patient-reported outcome measurements compared with placebo. ${ }^{5}$ Dapoxetine also was effective in men with lifelong and acquired PE.

Despite these favorable outcomes, treatment discontinuation rates are high. An integrated analysis of clinical dapoxetine trials 
Table 1. Patient baseline characteristics and discontinuation rates

\begin{tabular}{|c|c|c|c|c|c|c|}
\hline & $1 \mathrm{mo}, \mathrm{n}(\%)$ & $3 \mathrm{mo}, \mathrm{n}(\%)$ & $6 \mathrm{mo}, \mathrm{n}(\%)$ & $1 y, n(\%)$ & $2 y, n(\%)$ & Total, n (\%) \\
\hline \multicolumn{7}{|l|}{ PE type* } \\
\hline Acquired $(n=112)$ & $34(30.3)$ & 50 (44.6) & $16(14.3)$ & $4(3.6)$ & $2(1.8)$ & 106 (94.6) \\
\hline Lifelong $(n=70)$ & $14(20)$ & $14(20)$ & 16 (22.9) & $11(15.7)$ & $3(4.3)$ & 58 (82.9) \\
\hline \multicolumn{7}{|l|}{ IELT* } \\
\hline$>2 \min (n=72)$ & 25 (34.7) & 25 (34.7) & 14 (19.4) & 7 (9.7) & $0(0)$ & 71 (98.6) \\
\hline$<2 \min (n=110)$ & 23 (20.9) & 39 (35.5) & $18(16.4)$ & $8(7.3)$ & $5(4.5)$ & $93(84.5)$ \\
\hline \multicolumn{7}{|l|}{ Age } \\
\hline$>50$ y $(n=92)$ & $28(30.4)$ & $32(34.8)$ & $18(19.6)$ & $5(5.4)$ & $1(1.1)$ & $84(91.3)$ \\
\hline$<50$ y $(n=90)$ & $20(22.2)$ & $32(35.5)$ & 14 (15.6) & $10(11.1)$ & $4(4.4)$ & $80(88.9)$ \\
\hline \multicolumn{7}{|l|}{ PDE5 inhibitor status* } \\
\hline Users (n = 122) & 39 (31.9) & 50 (40.9) & $20(16.4)$ & $5(4.1)$ & $1(0.8)$ & 115 (94.3) \\
\hline Non-users $(n=60)$ & $9(15)$ & $14(23.3)$ & $12(20)$ & $10(16.7)$ & $4(6.7)$ & $49(81.7)$ \\
\hline \multicolumn{7}{|l|}{ IIEF-EFD scores* } \\
\hline$\geq 26(n=62)$ & 11 (17.7) & $15(24.2)$ & $10(16.1)$ & $11(17.7)$ & $3(4.8)$ & $50(80.6)$ \\
\hline$<26(n=120)$ & 37 (30.8) & $49(40.8)$ & $22(18.3)$ & $4(3.3)$ & $2(1.7)$ & $114(95)$ \\
\hline Total $(n=182)$ & $48(26.4)$ & 64 (35.2) & 32 (17.6) & $15(8.2)$ & $5(2.7)$ & 164 (90.1) \\
\hline
\end{tabular}

IELT = intravaginal ejaculation latency time; IIEF-EFD = International Index of Erectile Function erectile function domain; PDE5 = phosphodiesterase type 5; $\mathrm{PE}=$ premature ejaculation.

${ }^{*} P<.05$ between two groups at 2 years.

showed that $30.4 \%$ of included subjects discontinued mostly because of a perceived lack of efficacy and for personal reasons. ${ }^{5}$ We summarize the dapoxetine discontinuation rates, and the reasons for discontinuation, in recently published articles (Table 3). Although the discontinuation rate varied by study type and duration, it ranged from $1.5 \%$ to $89.6 \%$. In the Asia-Pacific Flexible Dose Study of Dapoxetine and Patient Satisfaction in Premature Ejaculation Therapy (PASSION) study by McMahon et $\mathrm{al}^{21}$ (a recent work in Asian-Pacific men with PE), more than $80 \%$ of subjects reported Clinical Global Impression of Change (CGIC) ratings of "slightly better" at 12 weeks. However, 23.5\% of subjects discontinued the drug.

The discontinuation rate in our study was higher than those noted in previous studies. We attribute this to differences in study design (study type and duration). In the phase 3 studies, patients were provided with the drug without cost, and the study durations did not exceed 12 weeks except in the work of Buvat et $\mathrm{al}^{16}$ (24 weeks; Table 3). Close monitoring, and the fact that the drug was free, might have enhanced compliance. In the real world, patients must pay for drugs and therefore would have high

Table 2. Reasons for discontinuation

\begin{tabular}{lc}
\hline Reason & $\mathrm{n}(\%)$ \\
\hline Cost & $49(29.9)$ \\
$\begin{array}{l}\text { Disappointed that PE was not curable and that } \\
\quad \text { dapoxetine was always required before sex }\end{array}$ & $41(25)$ \\
Side effects & $19(11.6)$ \\
Poor efficacy & $16(9.8)$ \\
Seeking other treatment options & $9(5.5)$ \\
Unknown & $30(18.3)$ \\
\hline
\end{tabular}

$\mathrm{PE}=$ premature ejaculation. expectations of efficacy. Moreover, if the medication instructions provided by the pharmacist or the physician were inadequate, then inappropriate use was possible, decreasing drug efficacy. We suggest that these factors led to us recording a higher discontinuation rate than in phase 3 studies. Our finding is similar to those of two studies performed in real-world settings; they were of longer duration than the phase 3 trials. ${ }^{4,8}$ Mondaini et $\mathrm{al}^{4}$ reported that $20 \%$ of patients with lifelong PE decided not to start dapoxetine treatment and almost $90 \%$ of those who initiated treatment discontinued within 1 year. Jern et $\mathrm{al}^{8}$ evaluated dapoxetine and paroxetine discontinuation rates, and the prevalence of side effects, in a real-world setting and found that the dapoxetine discontinuation rate was $70.6 \%$.

Turning to the reasons for discontinuation, it was noteworthy that neither adverse events nor lack of efficacy were of major importance (Table 3), unlike what was noted in the phase 3 studies. ${ }^{9-11}$ Open-label observational studies (including ours) showed that high cost, disappointment in the need for continual treatment, and personal reasons were significant for discontinuation. ${ }^{4,8}$ In the present study, cost was the most common reason for discontinuation, followed by disappointment that PE was not a curable disease and that dapoxetine was needed whenever sexual intercourse was contemplated. For the cost of dapoxetine, patients in South Korea cannot be covered by national health insurance for the cost of treating sexual disorders such as ED and PE. Dapoxetine 30 and $60 \mathrm{mg}$ are sold at approximately US $\$ 5.00$ and US\$10.00, respectively, which is similar to the price of PDE5 inhibitors. For the patient's disappointment, we suggest that physicians provide comprehensive counseling at the time of treatment commencement. Patients must understand that dapoxetine helps them to control ejaculation only temporarily and that $\mathrm{PE}$ is controllable and not curable. Careful 
Table 3. Comparison of prior studies with the present study

\begin{tabular}{|c|c|c|c|c|c|}
\hline Study & Study description & $\begin{array}{l}\text { Treatment } \\
\text { duration }\end{array}$ & Patients, $\mathrm{n}$ & $\begin{array}{l}\text { Discontinuation } \\
\text { rate, \% }\end{array}$ & Reasons for discontinuation (\%) \\
\hline Safarinejad ${ }^{23}$ (2008) & $\begin{array}{l}\text { double-blinded, } \\
\text { placebo-controlled, } \\
\text { fixed-dose, randomized }\end{array}$ & $12 \mathrm{wk}$ & 212 & 12.3 & $\begin{array}{l}\text { adverse events, lack of efficacy, lost to } \\
\text { follow-up }\end{array}$ \\
\hline \multirow[t]{2}{*}{ Buvat et $\mathrm{al}^{16}$ (2009) } & $\begin{array}{l}\text { randomized, double-blinded, } \\
\text { placebo-controlled phase } 3\end{array}$ & $24 w k$ & 1,162 & $43(30 \mathrm{mg})$ & $\begin{array}{l}\text { Subject's choice (21), lost to follow-up (6), } \\
\text { adverse event (4), other (12) }\end{array}$ \\
\hline & & & & 47 (60 mg) & $\begin{array}{l}\text { Subject's choice (21), adverse event (8), } \\
\text { lost to follow-up (5), other (12) }\end{array}$ \\
\hline Kaufman et $\mathrm{al}^{17}$ (2009) & $\begin{array}{l}\text { randomized, double-blinded, } \\
\text { placebo-controlled, phase } 3\end{array}$ & 9 wk & 1,238 & 10 & side effects \\
\hline McMahon et al ${ }^{25}$ (2010) & $\begin{array}{l}\text { phase } 3 \text { double-blinded, } \\
\text { parallel-group }\end{array}$ & $12 \mathrm{wk}$ & 1,067 & $\begin{array}{l}1.7(30 \mathrm{mg}) \\
5.1(60 \mathrm{mg})\end{array}$ & TEAEs \\
\hline Mondaini et $\mathrm{al}^{4}$ (2013) & prospective observational & $1 y$ & 120 & 89.6 & $\begin{array}{l}\text { below expectations }(24.4) \text {, cost }(22.1) \text {, side } \\
\text { effects (19.8), low interest in sex (19.8), } \\
\text { no efficacy (13.9) }\end{array}$ \\
\hline \multirow[t]{2}{*}{ Lee et $\mathrm{al}^{24}$ (2013) } & $\begin{array}{l}\text { prospective, randomized, } \\
\text { double-blinded, } \\
\text { placebo-controlled }\end{array}$ & $12 \mathrm{wk}$ & 57 (dapoxetine $30 \mathrm{mg}$ ) & 45.6 & $\begin{array}{l}\text { lost to follow-up (29.8), adverse event (8.8), } \\
\text { subject's choice (1.8), protocol violation (5.3) }\end{array}$ \\
\hline & & & $\begin{array}{l}63 \text { (dapoxetine } \\
30 \mathrm{mg}+\text { mirodenafil } \\
50 \mathrm{mg} \text { ) }\end{array}$ & 28.6 & $\begin{array}{l}\text { lost to follow-up (15.9), adverse event (7.9), } \\
\text { subject's choice (1.6), protocol violation (3.2) }\end{array}$ \\
\hline Mirone et $\mathrm{al}^{25}$ (2014) & open-label, observational & $12 \mathrm{wk}$ & 6,712 & 1.5 & side effect \\
\hline Simsek et $\mathrm{al}^{26}(2014)$ & open-label, observational & $1 \mathrm{mo}$ & 150 & 10 & $\begin{array}{l}\text { cost (5), side effect (3), effect below } \\
\text { expectation (2) }\end{array}$ \\
\hline Jiann and Huang ${ }^{27}$ (2015) & phase 4, open-label & $\begin{array}{l}\text { mean } 2.3 \pm 2.1 \\
\text { mo }(0-9)\end{array}$ & 314 & 25.7 & poor effectiveness (62.9), cost (45.7) \\
\hline Jern et $a^{8}(2015)$ & open-label, observational & mean 13.3 mo & 132 & 70.6 & side effects, limited efficacy, cost \\
\hline \multirow[t]{2}{*}{ Sahin et al ${ }^{28}(2016)$} & $\begin{array}{l}\text { prospective, randomized, } \\
\text { controlled }\end{array}$ & $1 \mathrm{mo}$ & 120 & $6.7(30 \mathrm{mg})$ & lost to follow-up (3.3), side effect (3.3) \\
\hline & & & & $10(60 \mathrm{mg})$ & side effect (6.7), lost to follow-up (3.3) \\
\hline Verze et al ${ }^{29}(2016)$ & $\begin{array}{l}\text { open-label, postmarketing } \\
\text { observational }\end{array}$ & $12 \mathrm{wk}$ & 6,128 & 10.9 & $\begin{array}{l}\text { lost to follow-up (3.5), personal reasons ( } 2.4) \text {, } \\
\text { insufficient response (1.6), adverse event (1.0) }\end{array}$ \\
\hline McMahon et al ${ }^{21}$ (2016) & open-label & $12 \mathrm{wk}$ & 285 & 23.5 & $\begin{array}{l}\text { withdrew consent (8.1), lost to follow-up (7.4), } \\
\text { adverse event (4.63) }\end{array}$ \\
\hline Present study & open-label, observational & $2 y$ & 182 & 90.1 & $\begin{array}{l}\text { cost (29.9), disappointment with on-demand } \\
\text { treatment (25), side effects (11.6), effect } \\
\text { below expectation (9.8), sought other options } \\
\text { (5.5), unknown (18.3) }\end{array}$ \\
\hline
\end{tabular}

TEAEs $=$ treatment-emergent adverse events. 
counseling along these lines might improve treatment compliance. Although we fully explained the mechanism of action of dapoxetine at the start of treatment, it seems that we did not prevent patients' disappointments during treatment.

Another interesting finding is that, although $79.1 \%$ of all patients discontinued treatment within 6 months, the discontinuation rate decreased sharply after 12 months. This is in agreement with the works of Mondaini et $\mathrm{al}^{4}$ and Jern et al, ${ }^{8}$ which were performed in real-world settings with follow-up times longer than 1 year. Jern et $\mathrm{al}^{8}$ reported that decisions to discontinue were usually made relatively soon after medication commenced; no patient discontinued medication after 30 months of usage. This emphasizes that, to improve compliance, it is essential that patients receive proper counseling, especially before starting treatment and/or in the early treatment period.

We found that patients with acquired PE (vs lifelong PE), with IELTs longer than 2 minutes before treatment, on PDE5 inhibitors, and with IIEF-EFD scores lower than 26 tended to exhibit high dropout rates at the end of the study. If patients with PE and ED on PDE5 inhibitors also took dapoxetine, the costs might have become too burdensome. Moreover, PDE5 inhibitors have been recently suggested to be useful treatments for PE. ${ }^{22}$ Thus, patients on PDE5 inhibitors might more readily stop dapoxetine treatment. However, unlike what we found, Jern et $\mathrm{al}^{8}$ reported that ED was more prevalent among those who continued dapoxetine treatment. Further study is needed on how concomitant PE and ED affect treatment of the other condition.

The high dropout rates of patients with acquired PE and baseline IELTs longer than 2 minutes might be attributable to the fact that their PE symptoms were not as severe as those of patients with lifelong PE and baseline IELTs shorter than 2 minutes; thus, it was easier for the former patients to stop their medication.

Although the dapoxetine discontinuation rate was very high, no SSRI withdrawal syndromes were noted. The common side effects were yawning, nausea, dizziness, and headache. These effects were mild and well tolerated.

To date, dapoxetine remains the only approved medical option for PE. No second-line therapy is available for patients who do not respond to dapoxetine or who refuse to take the drug. Thus, we performed a comprehensive evaluation of factors triggering dropout in real-world practice.

To the best of our knowledge, the present open-label observational study has the longest follow-up period (2 years) reported to date. However, several limitations should be noted. The study design meant that several biases were in play, the first of which was recall bias. We retrospectively collected data on adverse events and the reasons for discontinuation using the telephone or mail. Second, we included only patients who agreed to commence dapoxetine in a single center; we could not evaluate patients who decided not to start dapoxetine when our study was initiated. Therefore, our study population differed from those of the dapoxetine phase 3 trials. Thus, selection and response biases might have been in play.
However, we suggest that our study population was more similar to those encountered by physicians in real-world practice than the populations participating in phase 3 trials.

Although we identified the reasons for dapoxetine discontinuation, we did not analyze the associations between IELT changes and discontinuation rates. We suggest that treatment outcomes can affect compliance and the discontinuation rate. However, our study was purely observational in nature; we did not check the post-treatment IELTs of patients who discontinued treatment. We assessed five parameters: type of PE, baseline IELT, age, use of PDE5 inhibitors, and erectile function. Further studies assessing more parameters in multivariate analysis would be valuable.

\section{CONCLUSIONS}

In the present 2-year prospective observational study in a realworld setting, only $9.9 \%$ of patients continued treatment to 24 months; 79.1\% discontinued within 6 months. Patients with acquired PE (vs lifelong PE), with IELTs longer than 2 minutes before treatment, on PDE5 inhibitors, and with IIEF-EFD scores lower than 26 tended to exhibit high dropout rates. The principal reasons for discontinuation were cost and disappointment with the need for continual treatment, followed by side effects and perceived poor efficacy. These results indicate that a more comprehensive treatment strategy is needed to manage patients with PE.

Corresponding Author: Hyun Jun Park, Department of Urology, Pusan National University Hospital, 1-10, Ami-dong, Seo-gu Busan 602-739, Republic of Korea; E-mail: joon501@ naver.com

Conflicts of Interest: The authors report no conflicts of interest.

Funding: None.

\section{STATEMENT OF AUTHORSHIP}

\section{Category 1}

(a) Conception and Design Hyun Jun Park; Nam Cheol Park

(b) Acquisition of Data Tae Nam Kim; Seung Ryong Baek; Kyung Min Lee; Sangmin Choe

(c) Analysis and Interpretation of Data Hyun Jun Park; Sangmin Choe

\section{Category 2}

(a) Drafting the Article Hyun Jun Park; Nam Cheol Park

(b) Revising It for Intellectual Content Hyun Jun Park; Nam Cheol Park; Tae Nam Kim

\section{Category 3}

(a) Final Approval of the Completed Article Hyun Jun Park; Nam Cheol Park; Tae Nam Kim; Seung Ryong Baek; Kyung Min Lee; Sangmin Choe 


\section{REFERENCES}

1. Rowland D, Perelman M, Althof S, et al. Self-reported premature ejaculation and aspects of sexual functioning and satisfaction. J Sex Med 2004;1:225-232.

2. Althof SE, McMahon CG, Waldinger MD, et al. An update of the International Society of Sexual Medicine's guidelines for the diagnosis and treatment of premature ejaculation (PE). J Sex Med 2014;11:1392-1422.

3. McMahon CG. Current and emerging treatments for premature ejaculation. Sex Med Rev 2015;3:183-202.

4. Mondaini N, Fusco F, Cai T, et al. Dapoxetine treatment in patients with lifelong premature ejaculation: the reasons of a "Waterloo". Urology 2013;82:620-624.

5. McMahon CG, Althof SE, Kaufman JM, et al. Efficacy and safety of dapoxetine for the treatment of premature ejaculation: integrated analysis of results from five phase 3 trials. J Sex Med 2011;8:524-539.

6. Carvalheira AA, Pereira NM, Maroco J, et al. Dropout in the treatment of erectile dysfunction with PDE5: a study on predictors and a qualitative analysis of reasons for discontinuation. J Sex Med 2012;9:2361-2369.

7. Salonia A, Rocchini L, Sacca A, et al. Acceptance of and discontinuation rate from paroxetine treatment in patients with lifelong premature ejaculation. J Sex Med 2009; 6:2868-2877.

8. Jern P, Johansson A, Piha J, et al. Antidepressant treatment of premature ejaculation: discontinuation rates and prevalence of side effects for dapoxetine and paroxetine in a naturalistic setting. Int J Impot Res 2015;27:75-80.

9. De Amicis LA, Goldberg DC, LoPiccolo J, et al. Clinical followup of couples treated for sexual dysfunction. Arch Sex Behav $1985 ; 14: 467-489$.

10. Hawton K, Catalan J, Martin P, et al. Long-term outcome of sex therapy. Behav Res Ther 1986;24:665-675.

11. Andersson KE, Abdel-Hamid IA. Therapeutic targets for premature ejaculation. Maturitas 2011;70:26-33.

12. McCarty E, Dinsmore W. Dapoxetine: an evidence-based review of its effectiveness in treatment of premature ejaculation. Core Evid 2012;7:1-14.

13. Modi NB, Dresser MJ, Simon M, et al. Single- and multipledose pharmacokinetics of dapoxetine hydrochloride, a novel agent for the treatment of premature ejaculation. J Clin Pharmacol 2006;46:301-309.

14. Andersson KE, Mulhall JP, Wyllie MG. Pharmacokinetic and pharmacodynamic features of dapoxetine, a novel drug for 'on-demand' treatment of premature ejaculation. BJU Int 2006;97:311-315.

15. McMahon C, Kim SW, Park NC, et al. Treatment of premature ejaculation in the Asia-Pacific region: results from a phase III double-blind, parallel-group study of dapoxetine. J Sex Med 2010;7:256-268.

16. Buvat J, Tesfaye F, Rothman M, et al. Dapoxetine for the treatment of premature ejaculation: results from a randomized, double-blind, placebo-controlled phase 3 trial in 22 countries. Eur Urol 2009;55:957-967.

17. Kaufman JM, Rosen RC, Mudumbi RV, et al. Treatment benefit of dapoxetine for premature ejaculation: results from a placebo-controlled phase III trial. BJU Int 2009;103:651-658.

18. Pryor JL, Althof SE, Steidle C, et al. Efficacy and tolerability of dapoxetine in treatment of premature ejaculation: an integrated analysis of two double-blind, randomised controlled trials. Lancet 2006;368:929-937.

19. Jannini EA. Editorial comment on: Dapoxetine for the treatment of premature ejaculation: results from a randomized, double-blind, placebo-controlled phase 3 trial in 22 countries. Eur Urol 2009;55:967-968.

20. Porst H, McMahon CG, Althof SE, et al. Baseline characteristics and treatment outcomes for men with acquired or lifelong premature ejaculation with mild or no erectile dysfunction: integrated analyses of two phase 3 dapoxetine trials. J Sex Med 2010;7:2231-2242.

21. McMahon C, Lee SW, Kim SW, et al. The Asia-Pacific Flexible Dose Study of Dapoxetine and Patient Satisfaction in Premature Ejaculation Therapy: the PASSION study. Sex Med 2016;4:e18-e27.

22. Castiglione F, Albersen M, Hedlund P, et al. Current pharmacological management of premature ejaculation: a systematic review and meta-analysis. Eur Urol 2016;69:904-916.

23. Safarinejad MR. Safety and efficacy of dapoxetine in the treatment of premature ejaculation: a double-blind, placebo-controlled, fixed-dose, randomized study. Neuropsychopharmacology 2008;33:1259-1265.

24. Lee WK, Lee SH, Cho ST, et al. Comparison between on-demand dosing of dapoxetine alone and dapoxetine plus mirodenafil in patients with lifelong premature ejaculation: prospective, randomized, double-blind, placebo-controlled, multicenter study. J Sex Med 2013;10:2832-2841.

25. Mirone $V$, Arcaniolo D, Rivas D, et al. Results from a prospective observational study of men with premature ejaculation treated with dapoxetine or alternative care: the PAUSE study. Eur Urol 2014;65:733-739.

26. Simsek A, Kirecci SL, Kucuktopcu O, et al. Comparison of paroxetine and dapoxetine, a novel selective serotonin reuptake inhibitor in the treatment of premature ejaculation. Asian J Androl 2014;16:725-727.

27. Jiann BP, Huang YJ. Assessing satisfaction in men with premature ejaculation after dapoxetine treatment in real-world practice. Int J Clin Pract 2015;69:1326-1333.

28. Sahin S, Bicer M, Yenice MG, et al. A prospective randomized controlled study to compare acupuncture and dapoxetine for the treatment of premature ejaculation. Urol Int 2016;97:104-111.

29. Verze P, Cai T, Magno C, et al. Comparison of treatment emergent adverse events in men with premature ejaculation treated with dapoxetine and alternate oral treatments: results from a large multinational observational trial. J Sex Med 2016; 13:194-199. 